\title{
Value Distribution of Meromorphic Solutions and Their Derivatives of Complex Differential Equations
}

\begin{abstract}
Abdallah El Farissi
Faculty of Sciences and Technology, Bechar University, Bechar, Algeria

Correspondence should be addressed to Abdallah El Farissi; elfarissi.abdallah@yahoo.fr

Received 11 May 2013; Accepted 16 August 2013

Academic Editors: G. Ólafsson and C. Zhu

Copyright (c) 2013 Abdallah El Farissi. This is an open access article distributed under the Creative Commons Attribution License, which permits unrestricted use, distribution, and reproduction in any medium, provided the original work is properly cited.

We deal with the relationship between the small functions and the derivatives of solutions of higher-order linear differential equations $f^{(k)}+A_{k-1} f^{(k-1)}+\cdots+A_{0} f=0, k \geq 2$, where $A_{j}(z)(j=0,1, \ldots, k-1)$ are meromorphic functions. The theorems of this paper improve the previous results given by El Farissi, Belaïdi, Wang, Lu, Liu, and Zhang.
\end{abstract}

\section{Introduction and Statement of Result}

Throughout this paper, we assume that the reader is familiar with the fundamental results and the standard notations of Nevanlinna's value distribution theory (see $[1,2])$. In addition, we will use $\lambda(f)$ and $\lambda(1 / f)$ to denote, respectively, the exponents of convergence of the zero sequence and the pole sequence of a meromorphic function $f, \rho(f)$ to denote the order of growth of $f, \sigma(f)$ to denote the type of the entire function with $0<\rho(f)<\infty$, and $\bar{\lambda}(f)$ and $\bar{\lambda}(1 / f)$ to denote, respectively, the exponents of convergence of the sequence of distinct zeros and distinct poles of $f$. A meromorphic function $\varphi(z)$ is called a small function of a meromorphic function $f(z)$ if $T(r, \varphi)=o(T(r, f))$ as $r \rightarrow+\infty$, where $T(r, f)$ is the Nevanlinna characteristic function of $f$. In order to express the rate of growth of meromorphic solutions of infinite order, we recall the following definitions.

Definition 1 (see [2-4]). Let $f$ be a meromorphic function, and let $z_{1}, z_{2}, \ldots$, such that $\left(\left|z_{j}\right|=r_{j}, 0<r_{1} \leq r_{2} \leq \cdots\right)$, be the sequence of the fixed points of $f$, with each point being repeated only once. The exponent of convergence of the sequence of distinct fixed points of $f$ is defined by the following:

$$
\bar{\tau}(f)=\inf \left\{\tau>0: \sum_{j=1}^{+\infty}\left|z_{j}\right|^{-\tau}<+\infty\right\} .
$$

Clearly,

$$
\bar{\tau}(f)=\varlimsup_{r \rightarrow+\infty} \frac{\log \bar{N}(r, 1 /(f-z))}{\log r},
$$

where $\bar{N}(r, 1 /(f-z))$ is the counting function of distinct fixed points of $f(z)$ in $\{|z|<r\}$.

Definition 2 (see [4-6]). Let $f$ be a meromorphic function. Then the hyperorder $\rho_{2}(f)$ of $f(z)$ is defined by the following:

$$
\rho_{2}(f)=\varlimsup_{r \rightarrow+\infty} \frac{\log \log T(r, f)}{\log r} .
$$

Definition 3 (see $[4,5])$. Let $f$ be a meromorphic function. Then the hyperexponent of convergence of the sequence of distinct zeros of $f(z)$ is defined by the following:

$$
\bar{\lambda}_{2}(f)=\varlimsup_{r \rightarrow+\infty} \frac{\log \log \bar{N}(r, 1 / f)}{\log r},
$$

where $\bar{N}(r, 1 / f)$ is the counting function of distinct zeros of $f(z)$ in $\{|z|<r\}$.

For $k \geq 2$, we consider the following linear differential equation:

$$
f^{(k)}+A f=0,
$$


where $A(z)$ is a transcendental meromorphic function of finite order $\rho(A)=\rho>0$. Many important results have been obtained on the fixed points of general transcendental meromorphic functions for almost four decades (see [7]). However, there are a few studies on the fixed points of solutions of differential equations. In [8], Wang and Lü have investigated the fixed points and hyperorder of solutions of second-order linear differential equations with meromorphic coefficients and their derivatives, and they have obtained the following result.

Theorem A (see [8]). Suppose that $A(z)$ is a transcendental meromorphic function satisfying $\delta(\infty, A)=\lim _{r \rightarrow+\infty}(m$ $(r, A) / T(r, A))=\delta>0, \rho(A)=\rho<+\infty$. Then, every meromorphic solution $f(z)$ \# 0 of the equation

$$
f^{\prime \prime}+A(z) f=0
$$

satisfies that $f$ and $f^{\prime}, f^{\prime \prime}$ all have infinitely many fixed points and

$$
\begin{gathered}
\bar{\tau}(f)=\bar{\tau}\left(f^{\prime}\right)=\bar{\tau}\left(f^{\prime \prime}\right)=\rho(f)=+\infty, \\
\bar{\tau}_{2}(f)=\bar{\tau}_{2}\left(f^{\prime}\right)=\bar{\tau}_{2}\left(f^{\prime \prime}\right)=\rho_{2}(f)=\rho .
\end{gathered}
$$

Theorem A has been generalized to higher-order differential equations by Liu Ming-Sheng and Zhang Xiao-Mei as follows.

Theorem B (see [4]). Suppose that $k \geq 2$ and $A(z)$ are transcendental meromorphic functions satisfying $\delta(\infty, A)=$ $\delta>0, \rho(A)=\rho<+\infty$. Then every meromorphic solution $f(z) \not \equiv 0$ of (5) satisfies that $f$ and $f^{\prime}, f^{\prime \prime}, \ldots, f^{(k)}$ all have infinitely many fixed points and

$$
\begin{gathered}
\bar{\tau}(f)=\bar{\tau}\left(f^{\prime}\right)=\bar{\tau}\left(f^{\prime \prime}\right)=\cdots=\bar{\tau}\left(f^{(k)}\right)=\rho(f)=+\infty, \\
\bar{\tau}_{2}(f)=\bar{\tau}_{2}\left(f^{\prime}\right)=\bar{\tau}_{2}\left(f^{\prime \prime}\right)=\cdots=\bar{\tau}_{2}\left(f^{(k)}\right)=\rho_{2}(f)=\rho .
\end{gathered}
$$

In [9], Belaïdi and El Farissi extended the result of Theorem B, and they gave the following theorem.

Theorem C (see [9]). Suppose that $k \geq 2$ and $A(z)$ are transcendental meromorphic functions satisfying $\delta(\infty, A)=$ $\delta>0$ and $\rho(A)=\rho(0<\rho<+\infty)$. If $\varphi(z)$ \# 0 is a meromorphic function with finite $\operatorname{order} \rho(\varphi)<+\infty$, then every meromorphic solution $f(z) \neq \equiv$ of (5) satisfies

$$
\begin{aligned}
\bar{\lambda}(f-\varphi) & =\bar{\lambda}\left(f^{\prime}-\varphi\right)=\cdots=\bar{\lambda}\left(f^{(k)}-\varphi\right) \\
& =\rho(f)=+\infty, \\
\bar{\lambda}_{2}(f-\varphi) & =\bar{\lambda}_{2}\left(f^{\prime}-\varphi\right)=\cdots=\bar{\lambda}_{2}\left(f^{(k)}-\varphi\right) \\
& =\rho_{2}(f)=\rho .
\end{aligned}
$$

Recently, $\mathrm{Xu}$ et al. [10] investigated the relationship between small functions and the derivatives of solutions of the following equation:

$$
f^{(k)}+A_{k-1} f^{(k-1)}+\cdots+A_{0} f=0, \quad k \geq 2,
$$

and obtained the following theorems which improve the results given by Chen, Wang, Lu, Liu, Zhang, Belaïdi, and El Farissi.

Theorem D (see [10]). Let $k \geq 2$ and $A_{j}(j=0,1, \ldots, k-1)$ be entire functions with finite order and satisfy one of the following conditions:

(i) $\max \left\{\rho\left(A_{j}\right),(j=1, \ldots, k-1)\right\}<\rho\left(A_{0}\right)=\rho<+\infty$,

(ii) $0<\rho\left(A_{k-1}\right)=\rho\left(A_{k-2}\right)=\cdots=\rho\left(A_{0}\right)<\infty$ and $\max \left\{\sigma\left(A_{j}\right),(j=1, \ldots, k-1)\right\}=\sigma_{1}<\sigma\left(A_{0}\right)=\sigma ;$

Then, for every solution $f \neq \equiv \quad 0$ of (10) and for any entire function $\varphi \neq \equiv$ satisfying $\rho_{2}(\varphi)<\rho\left(A_{0}\right)$, one has the following:

$$
\bar{\lambda}_{2}\left(f^{(i)}-\varphi\right)=\lambda_{2}\left(f^{(i)}-\varphi\right)=\rho_{2}(f)=\rho, \quad i \in \mathbb{N} .
$$

Theorem E (see [10]). Let $k \geq 2$ and $A_{j}(j=0,1, \ldots, k-1)$ be meromorphic functions satisfying $\max \left\{\rho\left(A_{j}\right) \quad(j=1, \ldots, k-\right.$ $1)\}<\rho\left(A_{0}\right)=\rho<+\infty$ and $\delta\left(\infty, A_{0}\right)>0$. Then for every meromorphic solution $f \neq \equiv$ of (10) and for any meromorphic function $\varphi$ 非 0 satisfying $\rho_{2}(\varphi)<\rho\left(A_{0}\right)$, one has $\bar{\lambda}_{2}\left(f^{(i)}-\varphi\right)=$ $\lambda_{2}\left(f^{(i)}-\varphi\right) \geqslant \rho(i=0,1, \ldots)$.

In this paper, we will deal with the above equation, investigate the relationship between small functions and derivatives of solutions of (10), obtain some results, which improve the previous results given by $\mathrm{Xu}, \mathrm{Tu}$, and Zheng, and prove the following theorems.

Theorem 4. Let $k \geq 2$ and $A_{j}(j=0,1, \ldots, k-1)$ be meromorphic functions of finite order such that all solutions of $(10)$ satisfy $\rho(f)=+\infty$ and $\rho_{2}(f)=\rho$. Then if $\varphi(z) \not \equiv 0$ is a meromorphic function with $\rho_{2}(\varphi)<\rho$, then every solution $f(z) \not \equiv 0$ of (10) satisfies

$$
\begin{array}{ll}
\bar{\lambda}\left(f^{(i)}-\varphi\right)=\lambda\left(f^{(i)}-\varphi\right)=\rho(f)=+\infty, & i \in \mathbb{N}, \\
\bar{\lambda}_{2}\left(f^{(i)}-\varphi\right)=\lambda_{2}\left(f^{(i)}-\varphi\right)=\rho_{2}(f)=\rho, & i \in \mathbb{N} .
\end{array}
$$

Theorem 5. Let $k \geq 2$ and $A_{j}(j=0,1, \ldots, k-1)$ be meromorphic functions of finite order such that all solutions of (10) satisfy $\rho(f)=+\infty$. Then if $\varphi(z) \neq \equiv 0$ is a meromorphic function with $\rho(\varphi)<\infty$, then every solution $f(z) \neq \equiv$ of $(10)$ satisfies (12). Furthermore, if $\rho_{2}(f) \geqslant \rho$, and $\rho_{2}(\varphi)<\rho$, then

$$
\bar{\lambda}_{2}\left(f^{(i)}-\varphi\right)=\lambda_{2}\left(f^{(i)}-\varphi\right)=\rho_{2}(f) \geqslant \rho, \quad i \in \mathbb{N} .
$$

Remark 6. The following example shows that Theorem 4 is not valid when (10) has a finite order solution.

Example 7. For the following equation

$$
f^{\prime \prime}+\frac{e^{2 z}+e^{z}-1}{1-e^{z}} f^{\prime}+\frac{-e^{2 z}}{1-e^{z}} f=0
$$

we can easily get that $(*)$ has solutions $f_{1}(z)=e^{e^{z}}+\alpha e^{z}, \alpha \epsilon$ $\mathbb{C}-\{0\}$, and $f_{2}(z)=e^{z}$. Solution $f_{1}$ satisfies $\rho\left(f_{1}\right)=+\infty$ and $\rho_{2}(f)=1$. Take $\varphi(z)=\alpha e^{z}$; then $\rho_{2}(\varphi)<1$. Thus, we can get that $\bar{\lambda}\left(f^{\prime}-\varphi\right)=0 \neq \infty=\rho(f)$ and $\bar{\lambda}_{2}\left(f^{\prime}-\varphi\right)=0 \neq 1=\rho_{2}(f)$. 
Remark 8. The proof of Theorem 4 is quite different from that of Theorem D (see [10]). The main ingredient in the proof is Lemma 17.

Corollary 9. Under the assumptions of Theorem 4, if $\varphi(z)=$ $z$, then for every solution $f$ of (10), one has the following:

$$
\begin{gathered}
\bar{\tau}\left(f^{(i)}\right)=\tau\left(f^{(i)}\right)=\rho(f)=+\infty, \quad i \in \mathbb{N}, \\
\bar{\tau}_{2}\left(f^{(i)}\right)=\tau_{2}\left(f^{(i)}\right)=\rho_{2}(f)=\rho\left(A_{0}\right)=\rho, \quad i \in \mathbb{N} .
\end{gathered}
$$

Remark 10. Theorem 4 is the improvement of Theorems A, B, $\mathrm{C}$, and $\mathrm{D}$, and Theorem 5 is the improvement of Theorem E.

Corollary 11. Let $k \geq 2$ and $h_{j}(z)(j=0,1, \ldots, k-1)\left(h_{j} \not \equiv\right.$ $0)$ be entire functions that have finite order such that $\rho=$ $\max \left\{\rho\left(h_{j}\right): j=0,1, \ldots, k-1\right\}<n$. Let $P_{j}(z)=a_{n, j} z^{n}+$ $a_{n-1, j} z^{n-1}+\cdots+a_{1, j} z+a_{0, j}$ bepolynomials, where $a_{n, j} \neq 0(j=$ $0,1, \ldots, k-1), a_{i, j}(j=0,1, \ldots, k-1) ; i=(0,1, \ldots, n-1)$ are complex numbers, and suppose that $\arg a_{n, j} \neq \arg a_{n, 0}$ or $a_{n, j}=c_{n, j} a_{n, 0}$, where $0<c_{n, j}<1, j \in\{1,2, \ldots, k-1\}$. If $\varphi(z) \neq \equiv$ is an entire function with $\rho_{2}(\varphi)<n$, then every solution $f(z)$ \# 0 of the equation

$$
\begin{gathered}
f^{(k)}+h_{k-1} e^{P_{k-1}(z)} f^{(k-1)}+\cdots+h_{s} e^{P_{s}(z)} f^{(s)} \\
+\cdots+h_{1} e^{P_{1}(z)} f^{\prime}+h_{0} e^{P_{0}(z)} f=0
\end{gathered}
$$

satisfies (12) with $\rho=n$.

\section{Auxiliary Lemmas}

The following lemmas will be used in the proofs of Theorem 4 and Corollary 11.

Lemma 12 (see [11]). Let $k \geq 2$ and $h_{j}(z)(j=0,1, \ldots, k-1)$ $\left(h_{j} \neq 0\right)$ be entire functions that have finite order such that $\rho=$ $\max \left\{\rho\left(h_{j}\right): j=0,1, \ldots, k-1\right\}<n$. Let $P_{j}(z)=a_{n, j} z^{n}+$ $a_{n-1, j} z^{n-1}+\cdots+a_{1, j} z+a_{0, j}$ be polynomials, where $a_{n, j} \neq 0$ $(j=0,1, \ldots, k-1), a_{i, j} \quad(j=0,1, \ldots, k-1 ; i=0,1, \ldots, n-1)$ are complex numbers, and suppose that $\arg a_{n, j} \neq \arg a_{n, 0}$ or $a_{n, j}=c_{n, j} a_{n, 0}$, where $0<c_{n, j}<1, j \in\{1,2, \ldots, k-1\}$. Then every solution of (15) of infinite order and $\rho_{2}(f)=n$.

Lemma 13 (see [3]). Let $A_{0}, A_{1}, \ldots, A_{k-1}, F(\not \equiv 0)$ be finite order meromorphic functions. If $f$ is a meromorphic solution with $\rho(f)=+\infty$ of the following equation:

$$
f^{(k)}+A_{k-1} f^{(k-1)}+\cdots+A_{1} f^{\prime}+A_{0} f=F,
$$

then $\bar{\lambda}(f)=\lambda(f)=\rho(f)=+\infty$.

Lemma 14 (see [12]). Let $A_{0}, A_{1}, \ldots, A_{k-1}, F(\not \equiv 0)$ be finite order meromorphic functions. If $f$ is a meromorphic solution of (16), then we have the following statements:

(i) if $\max \left\{\sigma(F), \sigma\left(A_{j}\right)\right.$; $\left.j=0,1, \ldots, k-1\right\}<\sigma(f)=\sigma \leq$ $\infty$, then $\sigma(f)=\lambda(f)=\bar{\lambda}(f)$;

(ii) if $\max \left\{\sigma_{2}(F), \sigma\left(A_{j}\right)\right.$; $\left.j=0,1, \ldots, k-1\right\}<\sigma_{2}(f)=\sigma \leq$ $\infty$, then $\sigma_{2}(f)=\lambda_{2}(f)=\bar{\lambda}_{2}(f)$.
Let $A_{j}(j=0,1, \ldots, k-1)$ be meromorphic functions. We define the sequences of functions by the following:

$$
\begin{aligned}
& A_{j}^{0}=A_{j}, \quad j=0,1, \ldots, k-1, \\
& A_{k-1}^{i}=A_{k-1}^{i-1}+\frac{\left(A_{0}^{i-1}\right)^{\prime}}{A_{0}^{i-1}}, \quad i \in \mathbb{N}, \\
& A_{j}^{i}=A_{j}^{i-1}+A_{j+1}^{i-1} \frac{\left(\Psi_{j+1}^{i-1}\right)^{\prime}}{\Psi_{j+1}^{i-1}}, \quad j=0,1, \ldots, k-2, i \in \mathbb{N},
\end{aligned}
$$

where $\Psi_{j+1}^{i-1}=A_{j+1}^{i-1} / A_{0}^{i-1}$.

Remark 15. In the case where one of the functions of $A_{j}^{i}(j=$ $0,1, \ldots, k-1)$ is equal to zero, then $A_{j}^{i+1}=A_{j-1}^{i}(j=0,1$, $\ldots, k-1)$.

Lemma 16. Suppose that $f$ is a solution of (10). Then $g_{i}=f^{(i)}$ is a solution of the following equation:

$$
g^{(k)}+A_{k-1}^{i} g^{(k-1)}+\cdots+A_{0}^{i} g=0,
$$

where $A_{j}^{i}(j=0,1, \ldots, k-1)$ are given by (17).

Proof. Assume that $f$ is a solution of (10), and let $g_{i}=f^{(i)}$. We prove that $g_{i}$ is an entire solution of (18). Our proof is by induction. For $i=1$, differentiating both sides of (10), we obtain

$$
\begin{aligned}
f^{(k+1)} & +A_{k-1} f^{(k)}+\left(A_{k-1}^{\prime}+A_{k-2}\right) f^{(k-1)}+\cdots \\
& +\left(A_{1}^{\prime}+A_{0}\right) f^{\prime}+A_{0}^{\prime} f=0
\end{aligned}
$$

and replacing $f$ by

$$
f=-\frac{\left(f^{(k)}+A_{k-1} f^{(k-1)}+\cdots+A_{1} f^{\prime}\right)}{A_{0}},
$$

we get

$$
\begin{aligned}
f^{(k+1)} & +\left(A_{k-1}-\frac{A_{0}^{\prime}}{A_{0}}\right) f^{(k)} \\
& +\left(A_{k-1}^{\prime}+A_{k-2}-A_{k-1} \frac{A_{0}^{\prime}}{A_{0}}\right) f^{(k-1)} \ldots \\
& +\left(A_{1}^{\prime}+A_{0}-A_{1} \frac{A_{0}^{\prime}}{A_{0}}\right) f^{\prime}=0 .
\end{aligned}
$$

That is,

$$
g_{1}^{(k)}+A_{k-1}^{1} g_{1}^{(k-1)}+A_{k-2}^{1} g_{1}^{(k-2)} \cdots+A_{0}^{1} g_{1}=0 .
$$

Suppose that the assertion is true for the values which are strictly smaller than a certain $i$. We suppose $g_{i-1}$ is a solution of the following equation:

$$
g_{i-1}^{(k)}+A_{k-1}^{i-1} g_{i-1}^{(k-1)}+A_{k-2}^{i-1} g_{i-1}^{(k-2)}+\cdots+A_{0}^{i-1} g_{i-1}=0 .
$$


Differentiating (23), we can write the following:

$$
\begin{gathered}
g_{i-1}^{(k+1)}+A_{k-1}^{i-1} g_{i-1}^{(k)}+\left(\left(A_{k-1}^{i-1}\right)^{\prime}+A_{k-2}\right) g_{i-1}^{(k-1)}+\cdots \\
+\left(\left(A_{1}^{i-1}\right)^{\prime}+A_{0}^{i-1}\right) g_{i-1}^{\prime}+A_{0}^{\prime} g_{i-1}=0 .
\end{gathered}
$$

In (24), replacing $g_{i-1}$ by

$$
\begin{aligned}
& g_{i-1} \\
& =-\frac{\left(g_{i-1}^{(k)}+A_{k-1}^{i-1} g_{i-1}^{(k-1)}+A_{k-2}^{i-1} g_{i-1}^{(k-2)}+\cdots+A\left(g_{i-1}\right)^{\prime}\right)}{A_{0}^{i-1}}
\end{aligned}
$$

yields the following:

$$
\begin{aligned}
g_{i-1}^{(k+1)}+ & \left(A_{k-1}^{i-1}-\frac{\left(A_{0}^{i-1}\right)^{\prime}}{A_{0}^{i-1}}\right) g_{i-1}^{(k)} \\
& +\left(\left(A_{k-1}^{i-1}\right)^{\prime}+A_{k-2}-A_{k-1}^{i-1} \frac{\left(A_{0}^{i-1}\right)^{\prime}}{A_{0}^{i-1}}\right) g_{i-1}^{(k-1)}+\cdots \\
& +\left(\left(A_{1}^{i-1}\right)^{\prime}+A_{0}^{i-1}-A_{1}^{i-1} \frac{\left(A_{0}^{i-1}\right)^{\prime}}{A_{0}^{i-1}}\right) g_{i-1}^{\prime}=0 .
\end{aligned}
$$

That is,

$$
g_{i}^{(k)}+A_{k-1}^{i-1} g_{i}^{(k-1)}+A_{k-2}^{i-1} g_{i}^{(k-2)}+\cdots+A_{0}^{i-1} g_{i}=0 .
$$

Lemma 16 is thus proved.

Lemma 17. Let $A_{j}(j=0,1, \ldots, k-1)$ be meromorphic functions of finite order such that all solutions of (10) have infinite order and $\rho_{2}(f)=\rho$, and let $A_{j}^{i}(j=0,1, \ldots, k-1)$ be defined as in (17). Then the nontrivial meromorphic solution $g$ of the equation

$$
g^{(k)}+A_{k-1}^{i} g^{(k-1)}+\cdots+A_{0}^{i} g=0, \quad k \geq 2,
$$

satisfies $\rho(g)=+\infty$ and $\rho_{2}(g)=\rho$.

Proof. Let $\left\{f_{1}, f_{2}, \ldots, f_{k}\right\}$ be a fundamental system of solutions of (10). We show that $\left\{f_{1}^{(i)}, f_{2}^{(i)}, \ldots, f_{k}^{(i)}\right\}$ is a fundamental system of solutions of (28). By Lemma 16, it follows that $f_{1}^{(i)}, f_{2}^{(i)}, \ldots, f_{k}^{(i)}$ are solutions of (28). Let $\alpha_{1}, \alpha_{2}, \ldots, \alpha_{k}$ be constants such that

$$
\alpha_{1} f_{1}^{(i)}+\alpha_{2} f_{2}^{(i)}+\cdots+\alpha_{k} f_{k}^{(i)}=0 .
$$

Then, we have

$$
\alpha_{1} f_{1}+\alpha_{2} f_{2}+\cdots+\alpha_{k} f_{k}=P(z)
$$

where $P(z)$ is a polynomial of a degree less than $i$. Since $\alpha_{1} f_{1}+\alpha_{2} f_{2}+\cdots+\alpha_{k} f_{k}$ is a solution of (10), then $P$ is a solution of (10), and by the conditions of the lemma, we conclude that $P$ is an infinite order solution of (10); this leads to a contradiction. Therefore, $P$ is a trivial solution. We deduce that $\alpha_{1} f_{1}+\alpha_{2} f_{2}+\cdots+\alpha_{k} f_{k}=0$. Using the fact that $\left\{f_{1}, f_{2}, \ldots, f_{k}\right\}$ is a fundamental solution of (10), we get $\alpha_{1}=$ $\alpha_{2}=\cdots=\alpha_{k}=0$. Now, let $g$ be a nontrivial solution of (28). Then, using the fact that $\left\{f_{1}^{(i)}, f_{2}^{(i)}, \ldots, f_{k}^{(i)}\right\}$ is a fundamental solution of (28), we claim that there exist constants $\alpha_{1}, \alpha_{2}, \ldots, \alpha_{k}$ not all equal to zero, such that $g=$ $\alpha_{1} f_{1}^{(i)}+\alpha_{2} f_{2}^{(i)}+\cdots+\alpha_{k} f_{k}^{(i)}$. Let $h=\alpha_{1} f_{1}+\alpha_{2} f_{2}+\cdots+\alpha_{k} f_{k}$, $h$ be a solution of (10), and $h^{(i)}=g$. Hence, by conditions of the lemma, we get $\rho(h)=\rho(g)=+\infty$ and $\rho_{2}(h)=\rho_{2}(g)=$ $+\infty$.

Proof of Theorem 4. Assume that $f$ is a solution of (10). By the conditions of the theorem, we get $\rho(f)=\infty$ and $\rho_{2}(f)=\rho$. Taking $g_{i}=f^{(i)}$, then $\rho\left(g_{i}\right)=\infty$ and $\rho_{2}\left(g_{i}\right)=\rho$. Now, let $w(z)=g_{i}(z)-\varphi(z)$, where $\varphi$ is a meromorphic function with $\rho_{2}(\varphi)<\rho$.

Then $\rho(w)=\infty$ and $\rho_{2}(w)=\rho$.

In order to prove $\bar{\lambda}\left(g_{i}-\varphi\right)=\lambda\left(g_{i}-\varphi\right)=\infty$ and $\bar{\lambda}_{2}\left(g_{i}-\right.$ $\varphi)=\lambda_{2}\left(g_{i}-\varphi\right)=\rho$, we need to prove only that $\bar{\lambda}(w)=\lambda(w)=$ $\infty$ and $\bar{\lambda}_{2}(w)=\lambda_{2}(w)=\rho$. Using the fact that $g_{i}=w+\varphi$ and by Lemma 16 we get the following:

$$
\begin{aligned}
w^{(k)} & +A_{k-1}^{i} w^{(k-1)}+\cdots+A_{0}^{i} w \\
& =-\left(\varphi^{(k)}+A_{k-1}^{i} \varphi^{(k-1)}+\cdots+A_{0}^{i} \varphi\right)=F
\end{aligned}
$$

By $\rho\left(A_{j}^{i}\right)<\infty, \rho_{2}(\varphi)<\rho, \varphi \not \equiv 0$ and Lemma 17, we get $F \not \equiv$ 0 and $\rho_{2}(F)<\rho$. By Lemmas 13 and 14 we get $\bar{\lambda}(w)=\lambda_{2}(w)=$ $\rho(w)=\infty$ and $\bar{\lambda}_{2}(w)=\lambda_{2}(w)=\rho_{2}(w)=\rho$. The proof of Theorem 4 is complete.

Proof of Theorem 5. By the same reasoning as before we can prove Theorem 5.

Proof of Corollary 11. By Lemma 12, all solutions of (15) satisfy $\rho(f)=+\infty$ and $\rho_{2}(f)=n$. Applying Theorem 4 we get $\bar{\lambda}\left(f^{(i)}-\varphi\right)=\rho(f)=+\infty, i \in \mathbb{N}$ and $\bar{\lambda}_{2}\left(f^{(i)}-\varphi\right)=n, i \in \mathbb{N}$.

\section{Acknowledgment}

The author would like to thank the referee for his/her helpful remarks and suggestions to improve the paper. This paper is supported by ANDRU (Agence Nationale pour le Développement de la Recherche Universitaire) and University of Mostaganem (UMAB) (PNR Project Code 8/u27/ 3144).

\section{References}

[1] W. K. Hayman, Meromorphic Functions, Clarendon Press, Oxford, UK, 1964.

[2] R. Nevanlinna, Eindeutige Analytische Funktionen, Springer, Berlin, Germany, Zweite Auflage, Reprint, Die Grundlehren der mathematischen Wissenschaften, Band 46, 1974.

[3] Z. X. Chen, "The fixed points and hyper order of solutions of second order complex differential equations," Acta Mathematica Scientia, vol. 20, no. 3, pp. 425-432, 2000 (Chinese). 
[4] M. S. Liu and X. M. Zhang, "Fixed points of meromorphic solutions of higher order linear differential equations," Annales Academice Scientiarum Fennica, vol. 31, no. 1, pp. 191-211, 2006.

[5] J. Wang and H. X. Yi, "Fixed points and hyper order of differential polynomials generated by solutions of differential equation," Complex Variables, vol. 48, no. 1, pp. 83-94, 2003.

[6] H. X. Yi and C. C. Yang, The Uniqueness Theory of Meromorphic Functions, Science Press, Beijing, China, 1995 Chinese.

[7] Q. T. Zhang and C. C. Yang, The Fixed Points and Resolution Theory of Meromorphic Functions, Beijing University Press, Beijing, China, 1988 Chinese.

[8] J. Wang and W. R. Lü, "The fixed points and hyper-order of solutions of second order linear differential equations with meromorphic coefficients," Acta Mathematicae Applicatae Sinica, vol. 27, no. 1, pp. 72-80, 2004.

[9] B. Belaïdi and A. El Farissi, "Oscillation theory to some complex linear large differential equations," Annals of Differential Equations, vol. 25, no. 1, pp. 1-7, 2009.

[10] H. Y. Xu, J. Tu, and X. M. Zheng, "On the hyper exponent of convergence of zeros of $f^{(j)}-\phi$ of higher order linear differential equations," Advances in Difference Equations, vol. 2012, article 114, 16 pages, 2012.

[11] B. Belaïdi, "Some precise estimates of the hyper order of solutions of some complex linear differential equations," Journal of Inequalities in Pure and Applied Mathematics, vol. 8, no. 4, article 107, 14 pages, 2007.

[12] J. Tu and T. Long, "Oscillation of complex high order linear differential equations with coefficients of finite iterated order," Electronic Journal of Qualitative Theory of Differential Equations, no. 66, pp. 1-13, 2009. 


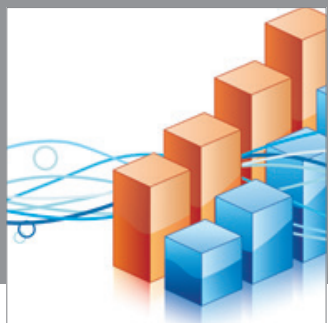

Advances in

Operations Research

mansans

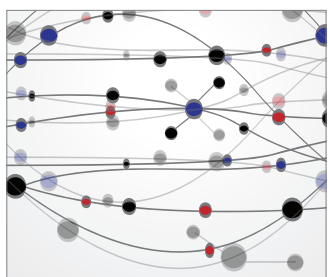

The Scientific World Journal
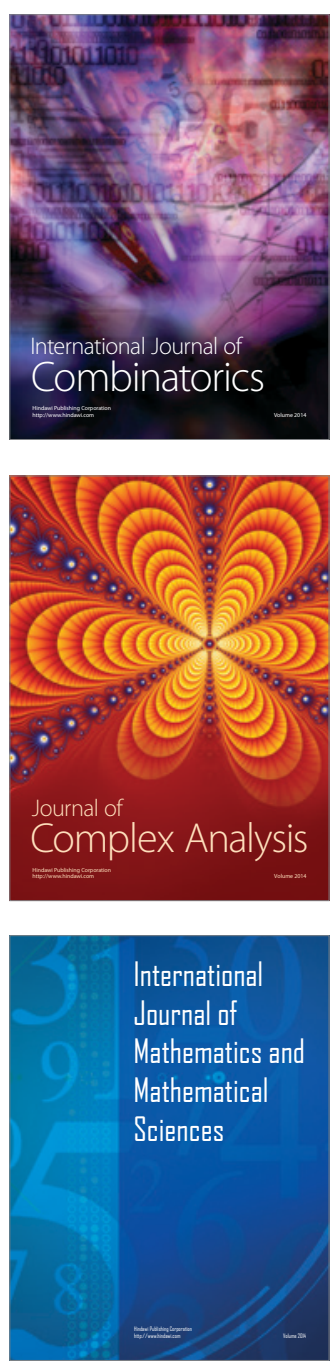
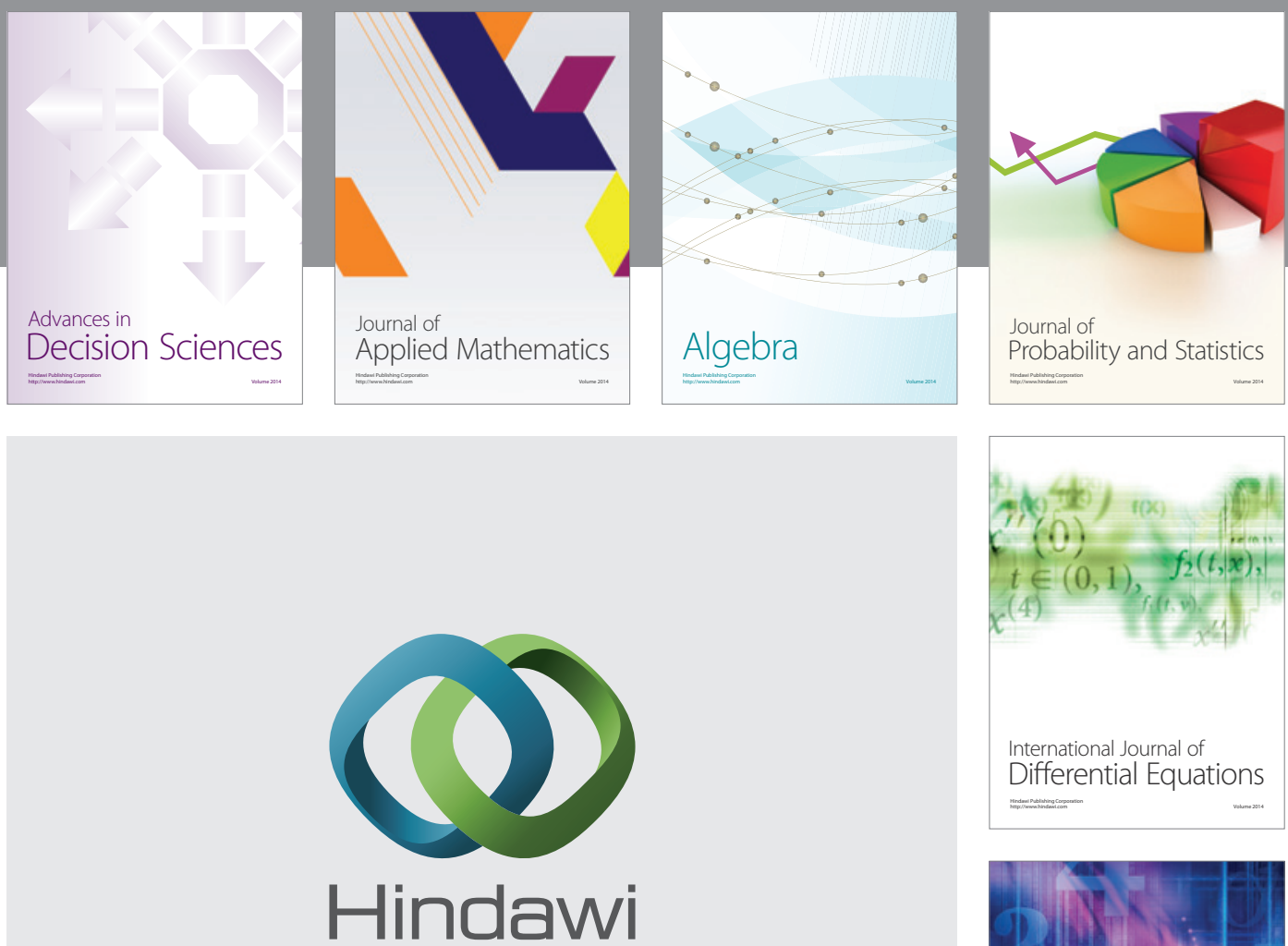

Submit your manuscripts at http://www.hindawi.com
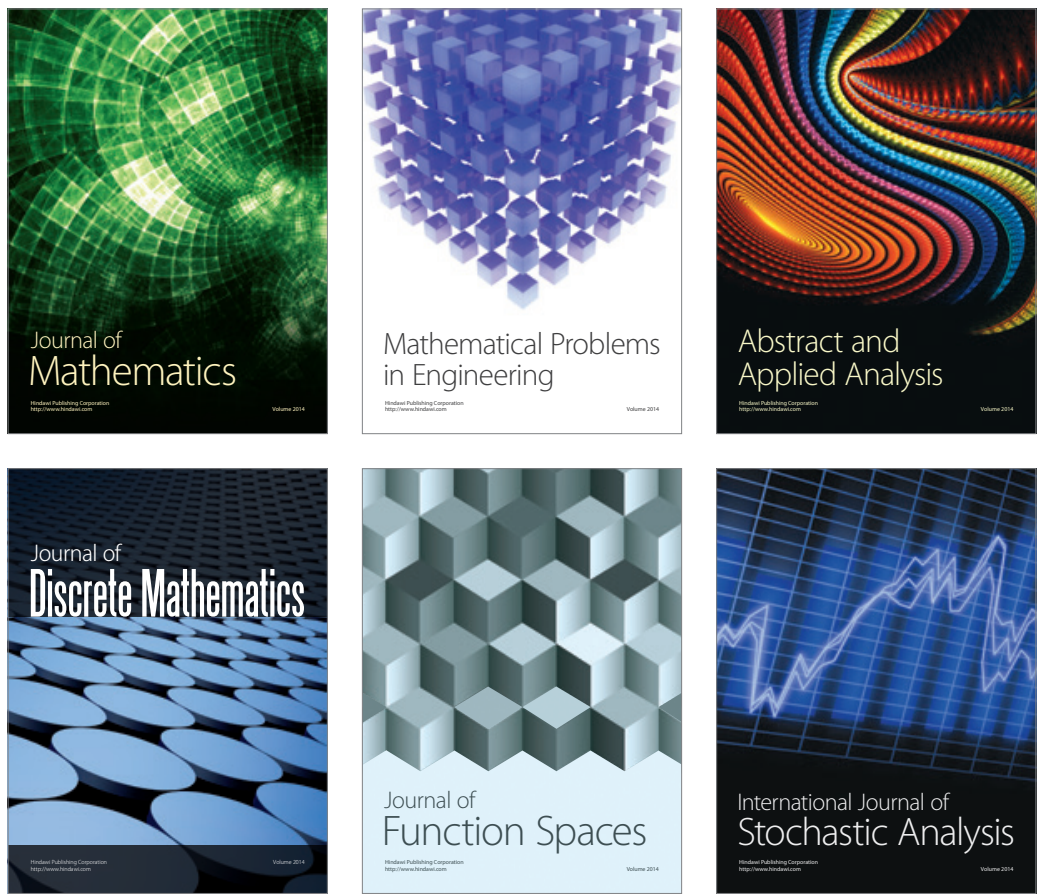

Journal of

Function Spaces

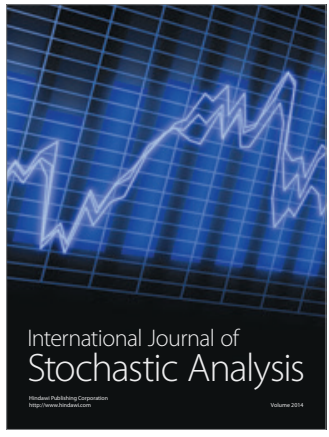

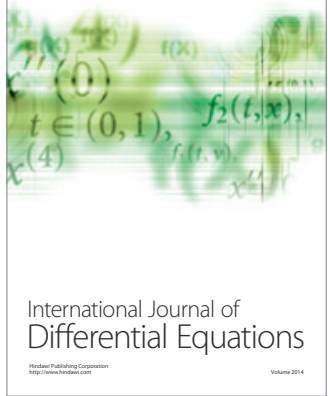
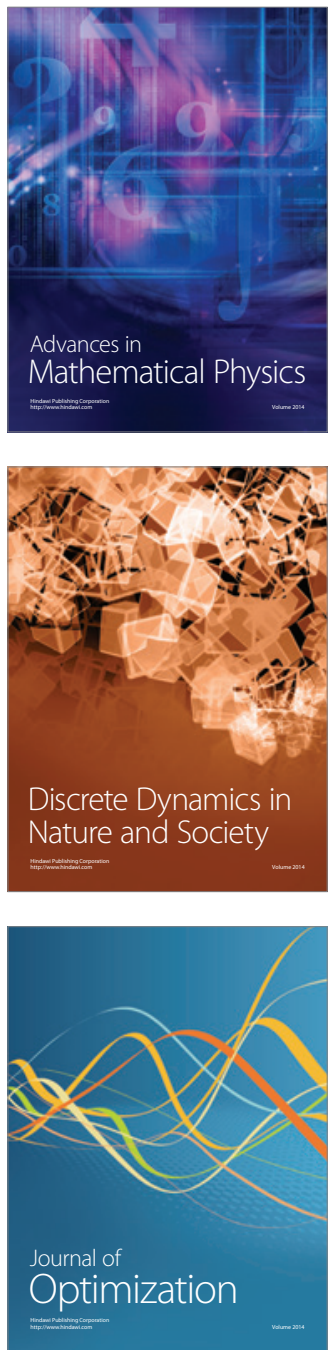